\title{
Optimum estimate of delays and dispersive effects in low-frequency interferometric observations
}

\author{
I. Martí-Vidal \\ Max-Planck-Institut für Radiastronomie, Auf dem Hügel 69, 53121 Bonn, Germany \\ e-mail: imartiv@mpifr.de
}

Received 23 December 2009 / Accepted 3 May 2010

\begin{abstract}
Context. Modern radio interferometers sensitive to low frequencies will make use of wide-band detectors (with bandwidths of the order of the observing frequency) and correlators with high data processing rates. It will be possible to simultaneously correlate data from many sub-bands spread through the whole bandwidth of the detectors. For such wide bandwidths, dispersive effects from the atmosphere introduce variations in the fringe delay which change through the whole band of the receivers. These undesired dispersive effects must be estimated and calibrated with the highest precision.

Aims. We studied the achievable precision in the estimate of the ionospheric dispersion and the dynamic range of the correlated fringes for different distributions of sub-bands in low-frequency and wide-band interferometric observations. Our study is focused on the case of sub-bands with a bandwidth much narrower than that of the total covered spectrum (case of LOFAR).

Methods. We computed the formal statistical uncertainty of the ionospheric delay, the delay ambiguity and the dynamic range of the correlated fringes using four different kinds of distributions of the sub-bands: constant spacing between sub-bands, random spacings, spacings based on a power-law distribution, and spacings based on Golomb rulers (sets of integers, $n_{i}$, whose sets of differences, $n_{j}-n_{i}$, have non-repeated elements).

Results. We compare the formal uncertainties in the estimate of ionospheric effects in the data, the ambiguity of the delays, and the dynamic range of the correlated fringes for the four different kinds of sub-band distributions.

Conclusions. For a large number of sub-bands ( $>20$, depending on the delay window) spacings based on Golomb rulers give the most precise estimates of dispersive effects and the highest dynamic ranges of the fringes. Spacings based on the power-law distribution give similar (but slightly worse) results, although the results are better than those from the Golomb rulers for a smaller numbers of sub-bands. Random distributions of sub-bands result in relatively large dynamic ranges of the fringes, but the estimate of dispersive effects through the band is worse. A constant spacing of sub-bands results in very bad dynamic ranges of the fringes, but the estimates of dispersive effects have a precision similar to that obtained with the power-law distribution. Combining all the results, the powerlaw distribution gives the best compromise between homogeneity in the sampling of the bandwidth, precision in the estimate of the ionospheric dispersive effects, dynamic range of the correlated fringes, and ambiguity of the group delay.
\end{abstract}

Key words. atmospheric effects - instrumentation: interferometers - techniques: interferometric - telescopes

\section{Introduction}

Modern radio interferometers sensitive to low frequencies, like the LOw Frequency ARray (LOFAR, see, e.g., de Vos, et al. 2009 and references therein), will make use of wide-band detectors (with a bandwidth of the order of the observing frequency) and correlators with a high data processing rate. For the case of LOFAR, it will be possible to correlate a bandwidth of $42 \mathrm{MHz}$, which can be divided into many sub-bands to be spread through a wide band (either 10-90 MHz, 110-190 MHz, 170-230 MHz, or 210-250 MHz; de Vos, et al. 2009). Therefore, the correlator will be able to simultaneously cover a large portion of the low-frequency spectrum. However, atmospheric dispersion may introduce strong frequency-dependent effects, which will be so different for each sub-band and must be estimated and calibrated with care. At low frequencies, the ionosphere is the main limiting factor in the quality of the interferometric observations. For the calibration of the ionospheric dispersion, the total electron content (TEC) must be estimated through the field of view (FoV) over each antenna and for each time. The TEC can be estimated, for instance, from global-positioning-system (GPS) data (e.g. Ros et al. 2000; Todorova, et al. 2006). With these techniques, however, it is not possible to obtain high-resolution estimates of the ionospheric turbulent screen over the FoV. For that purpose, it is necessary to apply self-calibration strategies, using therefore the interferometric data to derive the structure of the TEC screen over each station and its evolution (see Intema et al. 2009; Cohen \& Rottgering 2009, for a discussion on the ionospheric screens).

The distribution of sub-bands through the whole bandwidth of the detectors affects the scientific information that can be extracted from the observations, but is also important for a precise and accurate estimate of the atmospheric dispersion from the interferometric observables. The distribution of sub-bands has also an effect in delay space, since the interferometric fringes have a shape related to the Fourier transform of the bandpass (i.e., in our case, the distribution of sub-bands). Therefore, we should distribute the sub-bands in such a way that the spectral coverage and sampling are maximized, but the dynamic range of the fringes (i.e., the height of the fringe peak relative to that of the highest sidelobe) is also maximized to improve the sensitivity of the interferometer. Finding out the right distribution of sub-bands to achieve an optimum spectral sampling and fringe dynamic range is not that simple, and the answer may depend on 
each case, namely, the total bandwidth to be covered, the number of sub-bands, and the bandwidth of each sub-band. For instance, Petrachenko (2008) studied the performance of "broadband delays", which are computed from several bands (up to 5) spread from $2-3 \mathrm{GHz}$ to $11-14 \mathrm{GHz}$, as a function of the way these bands are distributed through the spectrum. Petrachenko (2008) concluded that the use of more than 2 bands, covering a total bandwidth as wide as possible, improves the performance of the interferometer.

In this paper, we report on a study of the spectral coverage, the precision in the estimate of atmospheric dispersive effects, the delay ambiguity, the dynamic range of the fringes, and the precision in the estimate of the source spectral index for different kinds of sub-band distributions and spectral configurations of an interferometer at low frequencies. The remainder of this paper is structured as follows: in Sect. 2 we describe the process of analysis. In Sect. 3 we describe the different sub-band distributions studied. In Sect. 4 we report on the results obtained and in Sect. 5 we summarize our conclusions.

\section{Analysis}

\subsection{Spectral configuration of the interferometer}

The spectral configuration of an interferometer can be characterized using the following parameters: i) minimum observing frequency, $v_{\mathrm{m}}$; ii) total bandwidth in units of the minimum observing frequency, i.e.,

$\beta=\left(v_{\mathrm{M}}-v_{\mathrm{m}}\right) / v_{\mathrm{m}}$,

where $v_{M}$ is the maximum observing frequency; iii) number of sub-bands, $N$ (and their distribution); and iv) the bandwidth of each sub-band, $\Delta v$. The $i$ th sub-band is centered at frequency $v_{i}$ (with $v_{\mathrm{m}}<v_{i}<v_{\mathrm{M}}$ ), which can be written as

$v_{i}=\left(1+\beta R_{i}\right) v_{\mathrm{m}}$,

being $R_{i}$ a real number between $0.5 \Delta v /\left(\beta v_{\mathrm{m}}\right)$ and $1-0.5 \Delta v /\left(\beta v_{\mathrm{m}}\right)$. The spectral configuration of an interferometric observation is then characterized by $v_{\mathrm{m}}, \beta, \Delta v_{i}$, and $R_{i}$. For simplicity, we assume the same $\Delta v_{i}=\Delta v$ for all sub-bands, and we also assume that $\Delta v \ll \beta v_{\mathrm{m}}$ (i.e., the bandwidth of the sub-bands is much narrower than the total covered bandwidth, so $R_{i}$ is defined between 0 and 1). This latter assumption corresponds to the case of the LOFAR interferometer.

\subsection{Dynamic range of the fringes}

The response of a baseline of an interferometer to a set of observed sources is equal to the addition of several fringes, one fringe for each source. The amplitude of these fringes is equal to a shrinked version of the amplitude of the Fourier transform of the bandpass (e.g., Thomson, et al. 1991). Spectral effects in the sources are not considered here. In the case of several subbands spread through a wider band, different shapes can be obtained for the fringes, which may have relatively large sidelobes. These large sidelobes may lead to confusion in the estimate of the fringe peaks. In frequency space, this effect can be understood as the possibility of fitting different slopes of phase vs. frequency to the same dataset. Minimizing the height of the sidelobes and maximizing their distance to the fringe peak decreases the probability of confusion and, therefore, enhances the sensitivity of the interferometer.
For each of the studied band distributions (described in Sect. 3), the shape of the fringes, $\boldsymbol{F}$, was computed as a vector with elements given by

$F_{k}=\left|\sum_{i=1}^{N} \cos \left(\pi \frac{v_{i}}{\beta v_{\mathrm{m}}}(k-1)\right)\right|$,

i.e., the module of the direct Fourier transform (DFT) of the subband distribution (the sine term of the DFT is zero). The vector $\boldsymbol{F}$ is thus computed assuming that the bandwidth of the sub-bands, $\Delta v$ is much narrower than the total bandwidth, $\beta v_{\mathrm{m}}$. The dynamic range of the fringe is estimated as the ratio between the fringe peak (which corresponds to the element $F_{1}$ ) and the peak of the highest sidelobe. We call this ratio $D$. The ambiguity of the delay is computed as the distance between these two peaks, in units of the Nyquist time resolution (i.e., $\left.1 /\left(2 \beta v_{\mathrm{m}}\right)\right)$. We call this quantity $\tau_{\mathrm{amb}}$. For the results reported in the following sections, we used a vector $\boldsymbol{F}$ of 512 elements.

If the width of the sub-bands is not much narrower than the total covered bandwidth, the dynamic range, $D$, is corrected by

$D \rightarrow D / \operatorname{sinc}\left(\pi \frac{\Delta v}{2 \beta v_{\mathrm{m}}} \tau_{\mathrm{amb}}\right)$

where $\operatorname{sinc}(x)=\sin (x) / x$.

\subsection{Estimate of the atmospheric dispersion}

The ionosphere introduces a change in the phase of the visibilities. For a given baseline and source, this phase depends on frequency as (e.g., Thomson, et al. 1991)

$\Delta \phi_{i}=K v_{i}^{-1}$.

The parameter $K$ is related to the TEC of the ionosphere over the elements of the baseline in the line-of-sight direction to the source. For a good calibration of the ionospheric dispersion, $K$ must be precisely estimated for each baseline, time, and pointing direction over the FoV of the interferometer.

The formal statistical uncertainty in the estimate of $K$ is that of the slope of the linear fit of $\phi_{i}$ vs. $v_{i}^{-1}$. It is straightforward to show that this uncertainty is

$\sigma(K) \propto\left(\left\langle v_{i}^{-2}\right\rangle-\left\langle v_{i}^{-1}\right\rangle^{2}\right)^{-1}=\sigma\left(v_{i}^{-1}\right)^{-1}$,

i.e., the precision in $K$ is proportional to the standard deviation of the distribution of the inverse of the central frequencies of the sub-bands, $\sigma\left(v_{i}^{-1}\right)$. This standard deviation maximizes when half of the sub-bands gather close to $v_{\mathrm{m}}$ and the other half gather close to $v_{\mathrm{M}}$. In this case

$\sigma\left(v_{i}^{-1}\right)_{\max }=0.5\left(v_{\mathrm{m}}^{-1}-v_{\mathrm{M}}^{-1}\right)$.

However, this distribution of sub-bands is not, by far, optimum, since the sampling of the total band is very poor and, moreover, spectral effects in the sources, which would not be well sampled through the bandwidth, could introduce important systematics in the estimate of the ionospheric dispersion using Eq. (5). Additionally, there could be several undetermined $2 \pi$-cycles of the phase drift caused by the ionosphere between $v_{\mathrm{m}}$ and $v_{\mathrm{M}}$, so, for this distribution, it would not be possible to connect the phases between all sub-bands to obtain a correct estimate of $K$.

The uncertainties in the estimate of the ionospheric dispersion, which we analyze in Sect. 4, were estimated as $\sigma(K)$ (computed from Eq. (6)) in units of its minimum possible value, $\sigma(K)_{\min }$ (i.e., that one corresponding to $\sigma\left(v_{i}^{-1}\right)_{\max }$, which is given in Eq. (7)). 


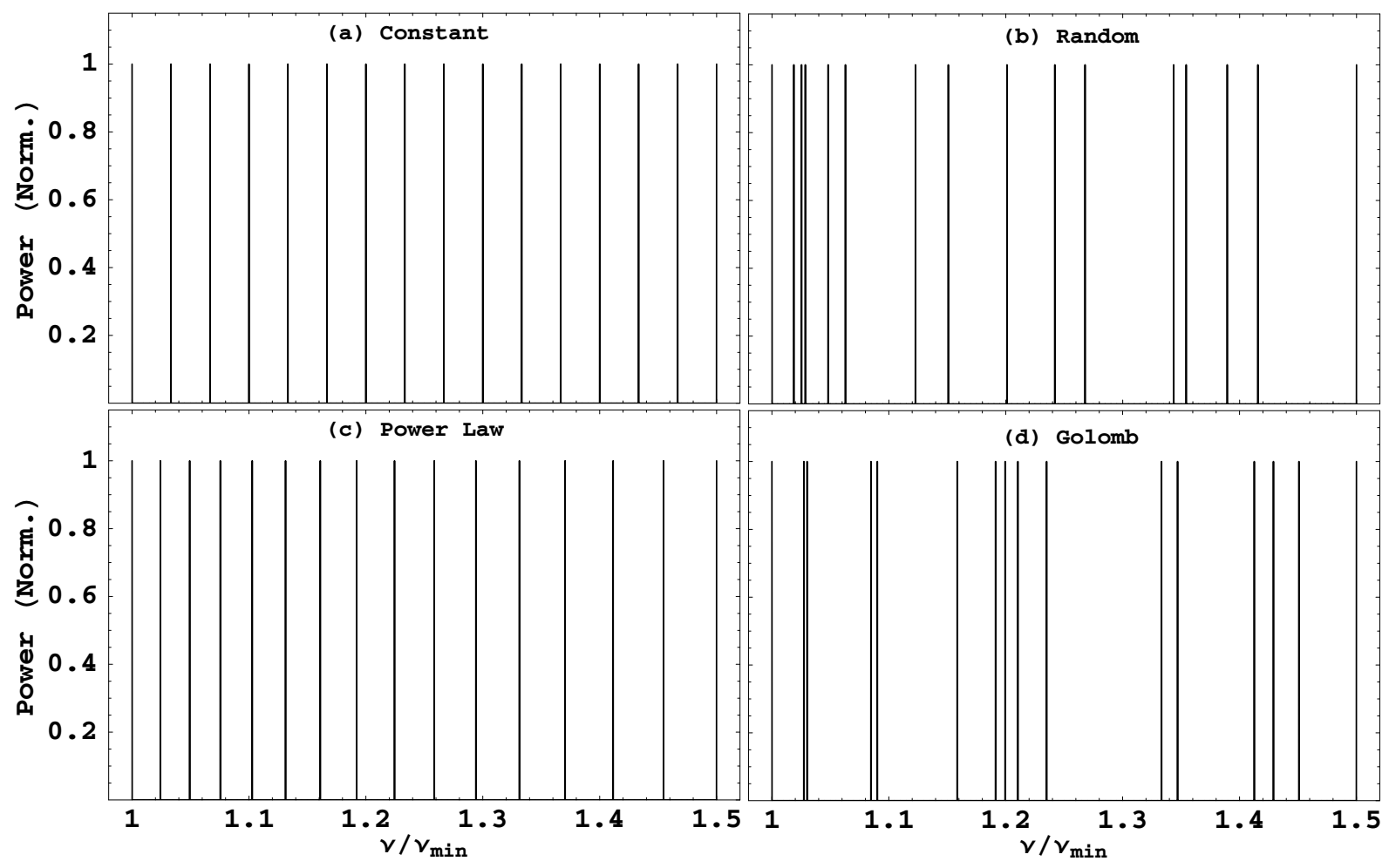

Fig. 1. Examples of the four kinds of distributions studied in this paper (using 16 sub-bands and setting $\beta=0.5$, see Eq. (1)). a) corresponds to the uniform (i.e. constant) distribution; b) to the random distribution; c) to the power-law distribution; and d) to the distribution based on the Golomb ruler.

\section{Distributions of sub-bands}

\subsection{Uniform (i.e., constant) distribution}

This is the most simple spectral configuration of the interferometer. The central frequencies of the sub-bands are distributed as

$v_{i}=\left(1+\beta \frac{i-1}{N-1}\right) v_{\mathrm{m}}$,

i.e., the frequency spacing between sub-bands is constant. We show an example of this distribution in Fig. 1a.

\subsection{Random distribution}

In this case, the sub-bands are randomly distributed over the bandwidth, i.e.,

$v_{i}=\left(1+\beta U_{i}\right) v_{\mathrm{m}}$,

where $U_{i}$ is a random real number between 0 and 1 , following a uniform statistical distribution. We show an example of this distribution of sub-bands in Fig. 1b. Different sets of $U_{i}$ may translate into different fringe dynamic ranges and precisions in the estimate of ionospheric dispersion (usually, a higher precision in the estimate of the atmospheric dispersion translates into a lower dynamic range of the fringes). We estimated the quantities $\sigma(K)$, $D$, and $\tau_{\text {amb }}$ as the averages of those computed from 100 different sets of $U_{i}$. Therefore, the results reported in Sect. 4 for the random distribution correspond to an averaged behavior of this distribution.

\subsection{Power-law distribution}

The ionosphere introduces larger phase drifts at lower frequencies. Therefore, it is plausible that a distribution that samples better the region of lower frequencies will give more precise estimates of the ionospheric dispersion, since the phase drifts will be better sampled in the region of the spectrum where the ionospheric effects are larger. A natural distribution to obtain this kind of sampling is setting the density of sub-bands proportional to a power law of frequency, $v^{\alpha}$, where $\alpha$ is a given (negative) constant, i.e.,

$\int_{v_{\mathrm{m}}}^{v_{i}} v^{\alpha} d v=\frac{i-1}{N-1} \int_{v_{\mathrm{m}}}^{v_{\mathrm{M}}} v^{\alpha} \mathrm{d} v$

Therefore,

$v_{i}=\left(\left(v_{\mathrm{M}}^{\alpha+1}-v_{\mathrm{m}}^{\alpha+1}\right) \frac{i-1}{N-1}+v_{\mathrm{m}}^{\alpha+1}\right)^{1 /(\alpha+1)}$.

For the special case of $\alpha=-1$ we have instead

$v_{i}=\exp \left(\left(\ln v_{\mathrm{M}}-\ln v_{\mathrm{m}}\right) \frac{i-1}{N-1}+\ln v_{\mathrm{m}}\right)$.

Equation (10) becomes Eq. (8) for $\alpha=0$. It can be shown that the standard deviation of $v_{i}^{-1}$ (i.e., the precision in the estimate of the ionospheric dispersion) is maximum for $\alpha=-5 / 3$ in the case of a large number of sub-bands. We show in Fig. 2 the optimum $\alpha$ as a function of the number of sub-bands, $N$. These values of $\alpha$ were computed by finding numerically the minimum of $\sigma(K)$ (using Eq. 6) as a function of $\alpha$. The values of $\alpha$ shown in Fig. 2 can be estimated using the model (which is also shown in the figure)

$\alpha=\frac{0.6}{N-4.35}-\frac{5}{3}$

which tends to $-5 / 3$ for large $N$. These are the values of $\alpha$ that were used to obtain the results reported in the next section. We show an example of this power-law distribution in Fig. 1c. 


\subsection{Golomb rulers}

A Golomb ruler is a set of $n_{i}$ integers such that the set of differences, $d_{i j}=n_{i}-n_{j}$, has no repeated elements (see, e.g., Atkinson, et al. 1986). It is intuitive that Golomb rulers are a good choice to maximize the dynamic range of the fringes, since all pairs of sub-bands are separated incoherently one respect to the other. Therefore, the sidelobes of the Fourier transform of the bandpass are minimum. The improvement in the fringe dynamic range when the sub-bands are distributed according to Golomb rulers has been previously reported for the case of 8 sub-bands (Mioduszewski \& Kogan 2004). Here we generalize the study to different number of sub-bands and also analyze the impact of this kind of distribution in the precision of the estimate of the ionospheric dispersion. The central frequencies, $v_{i}$, of the subbands are computed using the equation

$v_{i}=\left(1+\beta \frac{n_{i}}{n_{N}}\right) v_{\mathrm{m}}$,

where $n_{i}$ is the $i$ th element of a Golomb ruler of $N$ elements (by convention, $\left.n_{1}=0\right)$ ). The Golomb rulers for $N<24$ were taken from the OGR project at http: //distributed.net/ogr, and the others from Atkinson, Santoro \& Urrutia (1986). We show an example of this distribution in Fig. 1d.

\section{Results}

Figure 1 shows that the random and Golomb-rulers distributions tend to poorly sample some regions of the spectrum and oversample others. On the contrary, the constant and the power-law distributions sample the bandwidth in a more homogeneous way. A more homogeneous sampling of the spectrum is preferable to obtain information from as many regions of the bandwidth as possible. Moreover, a more homogeneous sampling makes easier the the connection of the phases between the sub-bands, since an unsampled wide lag in the spectrum could contain a number of $2 \pi$ phase cycles that could introduce biases in the data analysis. To better understand this statement, let us consider, for example, a non-dispersive delay, $\tau$, added to the fringe. The differential phase between sub-bands $i$ and $j$, due to $\tau$, would be

$\Delta \phi_{i j}=\phi_{j}-\phi_{i}=2 \pi \tau\left(v_{j}-v_{i}\right)$

For larger values of $v_{j}-v_{i}$ (i.e., for wider lags between sub-bands), the differential phase between sub-bands is larger. Therefore, the probability of the differential phase to be larger than $2 \pi$ is higher for wider lags between sub-bands.

From this point of view, the uniform and/or the power-law distributions would be the best frequency configurations for the interferometer. However, we must also take into account the dynamic range of the fringes and the quality in the estimate of the atmospheric dispersion, which are analyzed in the following subsections.

\subsection{Ionospheric dispersion}

In Fig. 3a, we show the uncertainty in the estimate of the ionospheric dispersion, $\sigma(K)$, in units of the minimum possible uncertainty (i.e., $\sigma(K)_{\min }$, computed from Eqs. (6) and (7)), as a function of the number of sub-bands, for a total bandwidth of $\beta=1.0$ (see Eq. (1)). The four different distributions are shown. For all distributions, the uncertainty (relative to the minimum possible one) increases with the number of sub-bands. It can be seen that the distribution based on Golomb rulers give the most

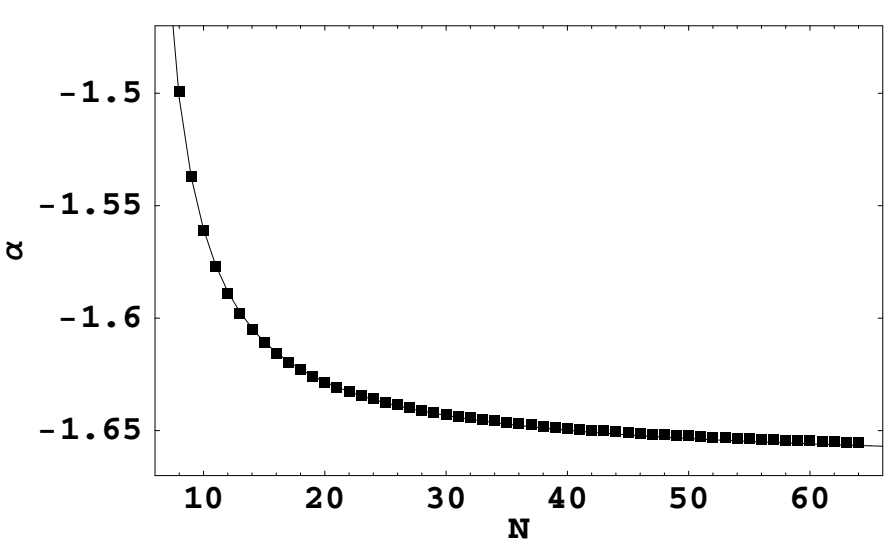

Fig. 2. Boxes, optimum values of $\alpha$ (i.e., those that minimize the formal uncertainty in the estimate of the ionospheric dispersion) as a function of the number of sub-bands. Line, model corresponding to Eq. (12).

precise estimates of the ionospheric dispersion, followed by the power-law distribution (with an uncertainty $\sim 3 \%$ larger, depending on the number of sub-bands), and the random and uniform distributions (with uncertainties $\sim 6 \%$ larger, also depending on the number of sub-bands).

In Fig. 4, we show the uncertainty in the estimate of the ionospheric dispersion, in units of the minimum possible uncertainty, as a function of the bandwidth (i.e., $\beta$, see Eq. (1)) using a total of 32 sub-bands to cover the bandwidth. Golomb rulers yield again the most precise estimates of the ionospheric dispersion, although the uncertainty increases as the bandwidth increases. On the contrary, the power-law distribution keeps the uncertainty roughly constant as a function of the bandwidth. Both the constant and random distributions also increase the uncertainty in the atmospheric dispersion as the bandwidth increases, being this uncertainty $\sim 5 \%$ larger than that obtained with the Golomb rulers.

We conclude that the power-law distribution and that based on Golomb rulers give higher precisions in the estimate of the ionospheric dispersion. Although the difference between uncertainties from all the distributions is not so large (lower than $10 \%$ in all cases), its optimization may be important to obtain highcontrast images.

\subsection{Fringe dynamic range and delay ambiguity}

In Fig. 3b, we show the dynamic range of the fringes as a function of the number of sub-bands for a total bandwidth of $\beta=1.0$ (see Eq. (1)). We notice, however, that the dynamic range of the fringes is independent of $\beta$, since a change in $\beta$ is equivalent to a change in the delay scaling of the fringes (regardless of a phase factor that depends on $v_{\mathrm{m}}$ ). Two regions in the sub-band space can be readily seen.

For $N<20$, the random and the power-law distributions give higher dynamic ranges. Suprisingly, for these values of $N$, the distribution based on Golomb rulers give dynamic ranges $\sim 1$. Why? Golomb rulers are sets of integer numbers. Therefore, the Fourier transforms of these sub-band distributions are periodic. If the delay window is larger than the period of the Fourier transform, there will be more than one peak in the fringe. The fringe period depends on each ruler and increases with the number of channels. For $N<20$, the fringe period is shorter than our delay window (1024 times the Nyquist time resolution, i.e., 512 channels in each direction of the delay) so there is more than one peak 


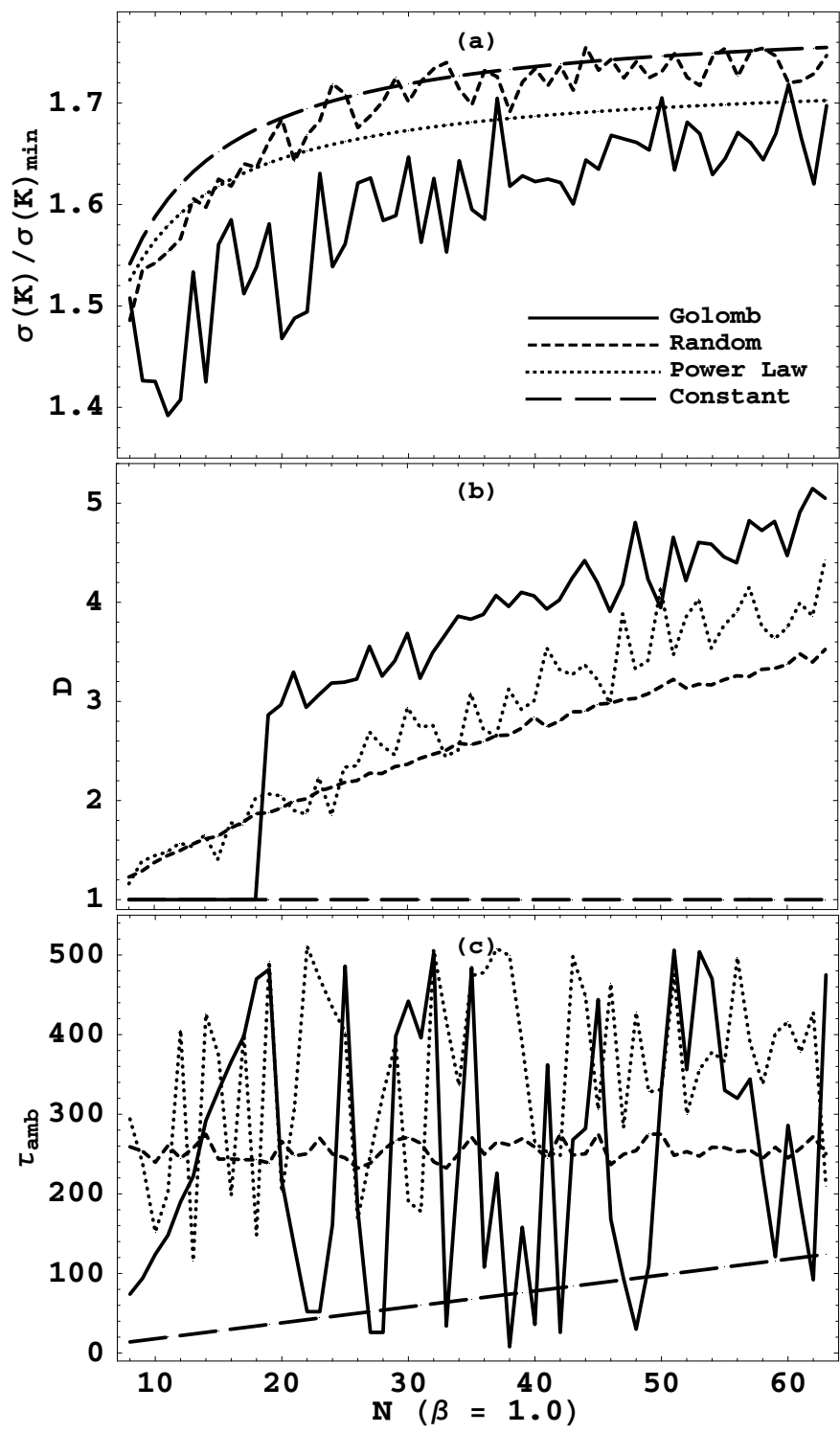

Fig. 3. a) Formal uncertainty in the estimate of the ionospheric dispersion, in units of the minimum possible uncertainty; b) dynamic range of the fringes; and c) delay ambiguity (i.e., distance between the fringe peak and the closest sidelobe) in units of the Nyquist time resolution. All these quantities are shown as a function of the number of sub-bands for $\beta=1.0$ (see Eq. (1)).

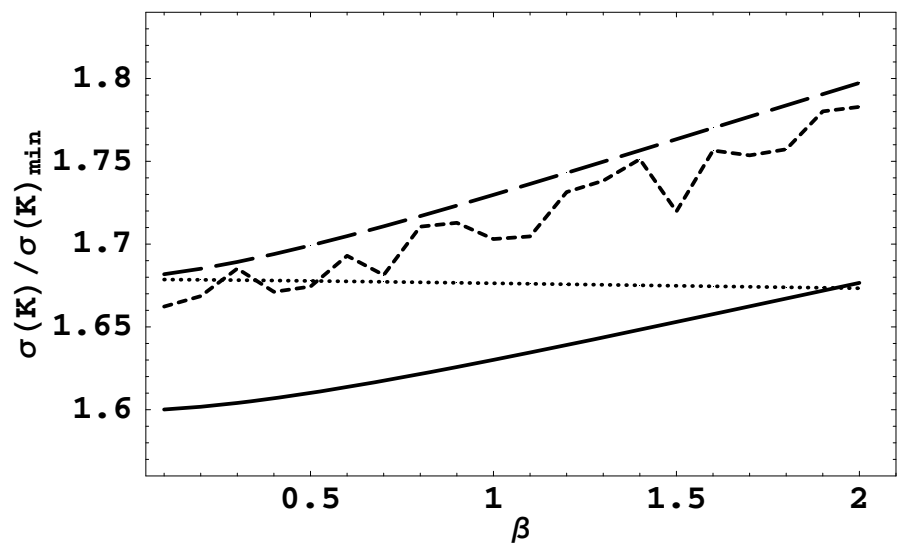

Fig. 4. Formal uncertainty in the estimate of the ionospheric dispersion, in units of the minimum possible uncertainty, as a function of $\beta$ (see Eq. (1)) for the case of 32 sub-bands. in the fringe. For $N>20$, the fringe period is larger and only one peak remains in the delay window. For $N>20$, Golomb rulers give the highest dynamic ranges (around 20-30\% higher than those based on the random and power-law distributions). In all cases, the uniform distribution gives very poor dynamic ranges, $\sim 1$, as it is indeed expected, since the Fourier transform of the bandpass is a periodic function with a very short period. For the case of the delay ambiguity, strong changes are seen as a function of the number of sub-bands for the Golomb rulers (the ambiguity ranges between $\sim 20$ and 512 channels) and the power-law distribution (the ambiguity ranges between $\sim 150$ and 512 channels, although the lower limit increases with $N$ ). These changes in the delay ambiguity are due to several sidelobes with similar peak values. Changing $N$ also changes the relative height of the sidelobe peaks. As a consequence, for different values of $N$, different sidelobes are selected as the highest and, therefore, very different delay ambiguities are obtained. On the contrary, the random distribution has a delay ambiguity of $\sim 250$ channels for all values of $N$ (we notice, however, that, for this distribution, the figure shows the average for 100 different fringes). The uniform distribution, as expected, has a very small delay ambiguity (lower than 100 channels). This ambiguity increases with $N$, also as expected, since the spacing of sub-bands (which is shorter for larger $N$ ) is inversely proportional to the period of the fringe.

A first conclusion is that the uniform distribution is not a good choice from the point of view of the quality in the estimate of the group delay. The Golomb rulers are a good choice when the number of sub-bands is large $(N>20$, although this number decreases if the width of the delay window decreases). The power-law distribution is, in general, a good choice for all $N$. It gives the best compromise between homogeneity in the sampling of the bandwidth, precision in the estimate of the ionsopheric dispersive effects, dynamic range of the correlated fringes, and ambiguity of the group delay. Therefore, this would be the preferable sub-band distribution to use in low-frequency (wide-band) interferometric observations.

Nevertheless, these conclusions are based on a number of sub-bands up to 64. If the number of sub-bands is large (say, $N=512$ ) there are no available Golomb rulers to work with, but we can still compare the results obtained from the uniform, random, and power-law distributions.

Setting $N=512$, the dynamic range of the fringes is similar for the three distributions, if we use a delay window of 1024 Nyquist channels (using 512 sub-bands, the period of the fringe corresponding to the uniform distribution is longer than the delay window). However, the power-law distribution still gives lower formal uncertainties in the estimate of the ionospheric dispersion (around 10\% lower than the other distributions for $\beta=2$ and $4 \%$ for $\beta=1$ ). Therefore, the power-law distribution is still the best choice with a number of sub-bands as large as 512 .

\subsection{Source spectral index}

Wide-band observations allow to precisely determine the spectral indices and spectral curvatures of radio sources. The different distributions of sub-bands may also affect the achievable precision in the estimate of the spectral properties of the radio sources. For the case of the spectral index, $\gamma$ (being the flux density, $\left.S \propto v^{\gamma}\right)$, the formal uncertainty, $\sigma(\gamma)$ depends on the distribution $v_{i}$ in the form

$\sigma(\gamma)=\sigma\left(\log v_{i}\right)^{-1}$ 


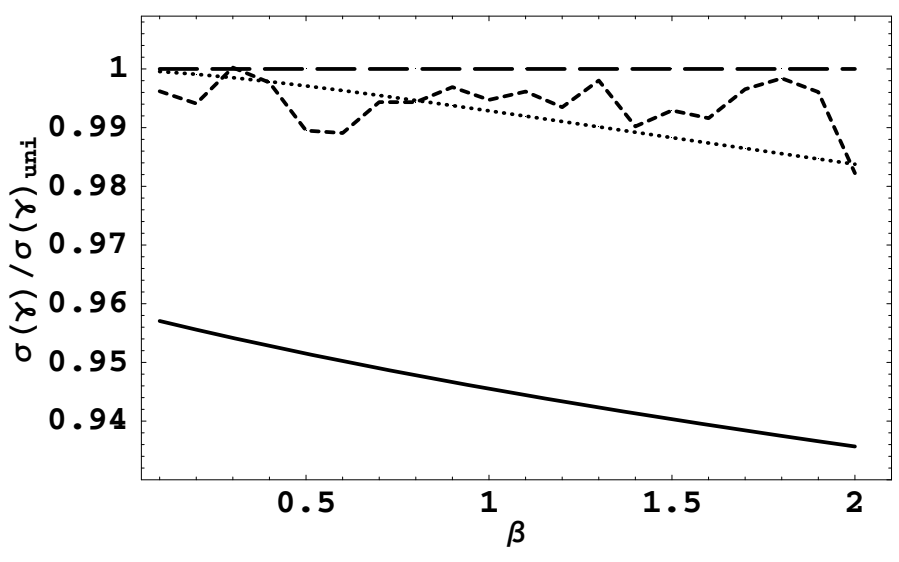

Fig. 5. Formal uncertainty in the estimate of the source spectral index, $\gamma$, in units of that of the uniform distribution $\left(\sigma(\gamma)_{\text {uni }}\right)$, as a function of the bandwidth, $\beta$ (see Eq. (1)), for the case of 64 sub-bands.

We show in Fig. 5 the formal uncertainty in the estimate of $\gamma$, in units of that of the uniform distribution, $\sigma(\gamma)_{\text {uni }}$, as a function of the bandwidth, $\beta$, for the case of 64 sub-bands. It can be seen in the figure that the uniform, random, and power-law distributions give similar precisions in the estimate of the spectral index (although the precision for the power-law distribution slightly increases with the bandwidth). Surprisingly, the Golomb-ruler distribution gives a precision $\sim 5 \%$ higher than the other distributions for all bandwidths.

The formal uncertainties of the uniform, random, and powerlaw distributions for a large number of sub-bands $(N=512)$ are similar to those shown in Fig. 5. Therefore, for wide-band observations using many sub-bands (i.e. where no Golomb rulers are available), the use of the power-law distribution is the best choice, allowing for a $1-2 \%$ higher precision in the estimate of the spectral index of the sources. With a smaller number of subbands, the use of Golomb-ruler distributions would improve the precision in the estimate of $\gamma$ by $\sim 5 \%$.

\subsection{Other contributions to the optimum power-law distribution}

Other contributions to the visibility phases (either due to the electron plasma of the ionosphere or to chromaticity in the structure of the observed sources), as well as the contribution of the galactic radiation to the visibility noise, have not been considered in the previous sections. In this section, we study how these contributions may affect the optimum sampling of the ionospheric dispersion using the power-law distribution of subbands.

\subsubsection{Plasma frequency of ionospheric electrons}

Equation (5) holds in the region of frequencies much higher than the plasma frequency, $v_{\mathrm{p}}$, of the ionospheric electrons. The electron density in the ionosphere takes values around $10^{4}-10^{6} \mathrm{e}^{-} \mathrm{cm}^{-3}$. This translates into a plasma frequency in the range $\sim 1-10 \mathrm{MHz}$ (e.g., Pacholczyk 1970, Eq. (2.72)). From the refraction index of a plasma (e.g., Pacholczyk 1970, Eqs. (2.77) and (2.78)), the phase drift in the case of $v_{i} \sim v_{\mathrm{p}}$ is

$\Delta \phi_{i}=K^{\prime} v_{i}\left(\sqrt{1+\frac{v_{\mathrm{p}}^{2}}{v_{i}\left(v_{i} \pm v_{\mathrm{B}}\right)}}-1\right)$, where $v_{\mathrm{B}}$ is the Larmor electron frequency (for the Earth magnetic field it takes the value $\sim 1 \mathrm{MHz}$ ) and the \pm signs correspond to the two possible circular polarizations of the radiation. We notice that the effect of $v_{\mathrm{B}}$ in the computations reported in this section is negligible. $K^{\prime}$ is related to the TEC above each element of the baseline in the line-of-sight to the source.

We computed the optimum values of $\alpha$ (i.e., the exponent of the power-law distribution of sub-bands) that optimize the sampling of the ionospheric phase drifts (i.e., minimize the formal uncertainty in the estimate of $K^{\prime}$ ) for observing frequencies close to $v_{\mathrm{p}}$. We call these values $\alpha_{\mathrm{p}}$, to distinguish them from the values without the effect of the plasma frequency (i.e., $\alpha$, which are shown in Fig. 2 and given in Eq. (12)). In Fig. 6 we show the ratios $\alpha_{p} / \alpha$ as a function of the minimum observing frequency, $v_{\mathrm{m}}$, in units of the plasma frequency, $\nu_{\mathrm{p}}$. For instance, using a bandwidth of $\beta=1.0$ (i.e., $v_{\mathrm{M}}=2 v_{\mathrm{m}}$ ) a plasma frequency $v_{\mathrm{p}}=10 \mathrm{MHz}$, and a minimum frequency $v_{\mathrm{m}}=100 \mathrm{MHz}$ (i.e, 10 times the plasma frequency) results in values of $\alpha_{p}$ equal to 0.86 times those of $\alpha$. For a large number of sub-bands (i.e., for $\alpha=-5 / 3$ ) this results in $\alpha_{\mathrm{p}}=-1.43$.

We notice that the exponent $\alpha_{p}$ approaches zero as the minimum frequency approaches to the plasma frequency. Therefore, the optimum power-law distribution approaches the constant distribution at very low frequencies. Increasing the bandwidth tends to compensate a bit the decrease in the absolute value of $\alpha_{p}$ (i.e., the ratios $\alpha_{p} / \alpha$ increase when $\beta$ increases) although this effect is small, as it can be appreciated in Fig. 6.

\subsubsection{Frequency-dependent source structure}

A source structure which is independent of the observing frequency is a strong assumption over the broad frequency ranges considered in the previous sections. The contribution of a possible source chromaticity in the visibility phases can be divided in two parts. One is related to the source being intrinsically different at different frequencies (this contribution might be especially important for extended sources) and the other one is related to the position of the source (or that of its brightest feature) being different at different frequencies (as it is the case, for instance, of a self-absorbed core-jet structure).

On the one hand, the contribution of the source structure to the visibility phases can be determined from the image of the source at each frequency. This image depends on the visibility calibration, but can also be used to refine such calibration. Therefore, it should be possible, in principle, to decouple the source structure (which introduces baseline-dependent phases) from the ionospheric dispersion (which introduces antennadependent phases), with the help of iterative and elaborated imaging-calibration algorithms. The details of these algorithms and their impact in the precision of the estimated ionospheric contribution, as it is decoupled from the source-structure contribution after the imaging, is beyond the scope of this paper.

On the other hand, the contribution of a frequency-dependent source position on the visibility phases can be studied if some assumptions are considered. Porcas (2009) reported on the effects of source chromaticity in the source position estimates through VLBI astrometry, performed using either phase delays or group delays, for the case of a core-jet structure following the model of Blandford \& Königl (1979). The contribution of the chromatic core-shift to the interferometric phase is

$\Delta \phi_{\text {str }}=K_{s} v^{1-\delta}$,

where $K_{s}$ depends on the physical conditions in the jet and the angle of the projected baseline with respect to that of the jet. 
I. Martí-Vidal: Optimum estimate of delays and dispersive effects

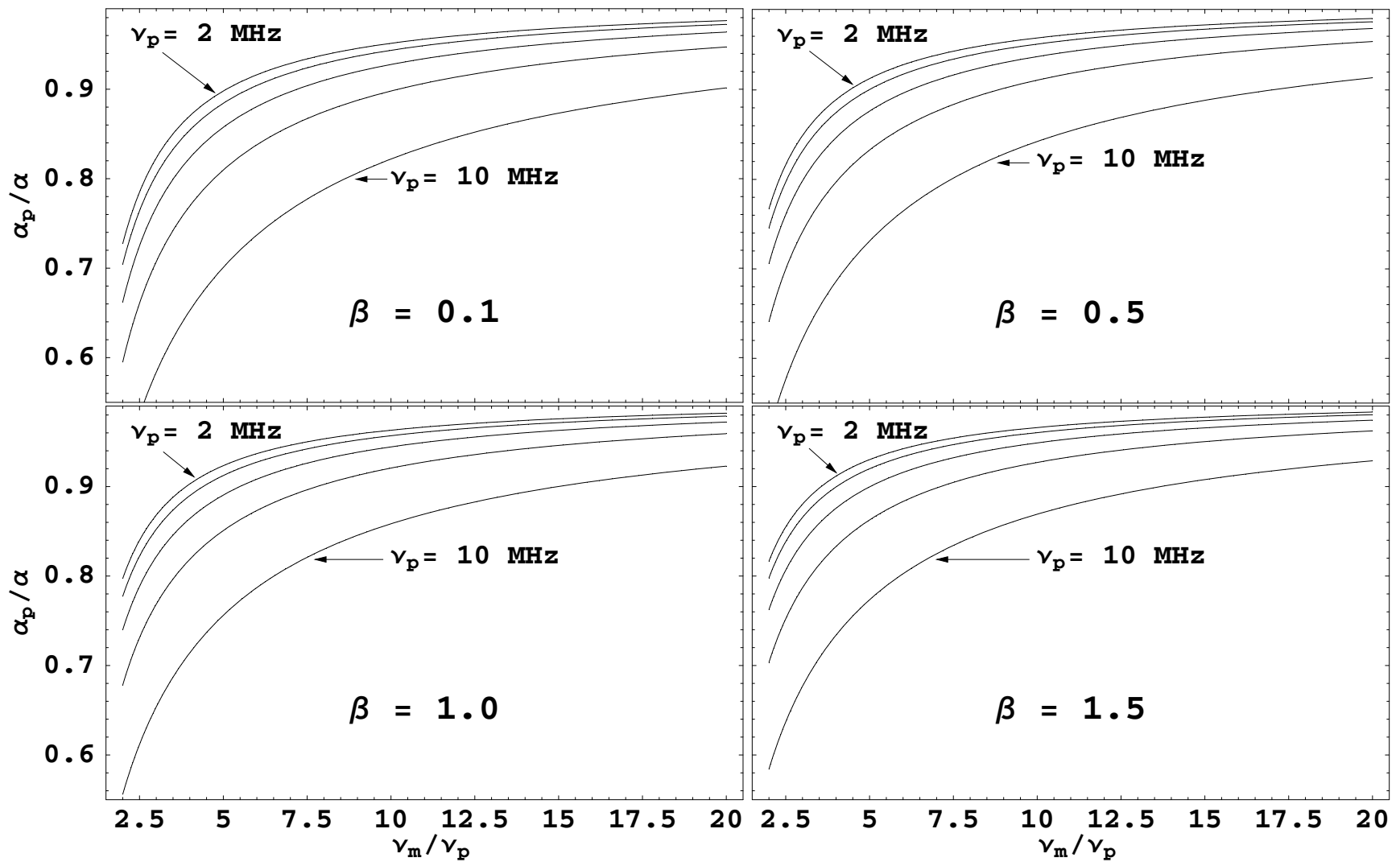

Fig. 6. Optimum values of $\alpha_{\mathrm{p}}$, in units of $\alpha$, as a function of the minimum observing frequency, $v_{\mathrm{m}}$, in units of the plasma frequency, $v_{\mathrm{p}}$. In each figure, the values are computed for five different values of $v_{\mathrm{p}}(2,4,6,8$, and $10 \mathrm{MHz})$. Each figure corresponds to a different bandwidth, $\beta(0.1$, $0.5,1.0$, and 1.5).

The parameter $\delta$ also depends on the physical conditions in the jet and may take values between 0 and $\sim 2$. If we combine Eqs. (5) and (16), we obtain the total drift of the visibility phases through the sub-bands when both effects, ionospheric dispersion and source chromaticity, are taken into account:

$\Delta \phi_{i}=\left(K+K_{s} v_{i}^{2-\delta}\right) v^{-1}$.

Therefore, the effect of a frequency-dependent source position is equivalent to the addition of an extra term coupled to the parameter $K$ of the ionospheric dispersion. For $\delta=0$ (i.e., no core-shift), $K_{s}$ translates into a contribution to $\phi$ equal to a constant (i.e., non-dispersive) group delay, with the phase proportional to the frequency. This group delay is equivalent to a shift of the source, which is the same at all frequencies. This shift can be easily fixed in the calibration if the source position is known. For $\delta=1$, the effect of the source chromaticity on $\phi$ is just adding a constant, so it does not affect $\sigma(K)$ (see Eq. (6)). Therefore, the sub-band distribution for the optimum estimate of ionospheric dispersion will be the same as with no chromatic effect. However, for the (physically unrealistic) case $\delta=2$, there is a complete coupling between the ionospheric dispersion, $K$, and the source chromaticity, $K_{s}$, so it is especially difficult to calibrate the ionospheric delay using these sources, regardless of the distribution of sub-bands used. Real sources may have values of $\delta$ falling between the values here analyzed ( 0,1 , and 2$)$. It is thus expected to find intermediate cases in which the effect of the frequency-dependent source position will be a combination of either a non-dispersive group delay, no effect in the estimate of ionospheric dispersion, and a complete coupling between that estimate and the source position. Additionally, all the core-jet sources detected in a given FoV may have different values of $\delta$, so different calibration issues will appear in the same image depending on the coordinates of each source and the values of $\delta$.

In any case, we notice that $K_{s}$ depends on the direction of the projected baseline relative to that of the jet, so it is a baselinedependent quantity. However, the ionospheric contribution, $K$, is antenna-dependent. This different behavior of $K_{s}$ and $K$, depending on the pair of stations selected, should allow for a robust decoupling of $K_{s}$ from $K$, provided the number of observing stations is large enough. Therefore, any chromaticity in the source structure and/or position should not affect the results reported in this paper, as long as the baseline-dependent chromatic effects are decoupled from the antenna-dependent ionospheric contribution using the appropriate calibration algorithms.

\subsubsection{Radiation from the Galaxy}

For frequencies below $\sim 400 \mathrm{MHz}$, the sky brightness temperature is dominated by the Galactic radiation, which depends strongly on the observing frequency $\left(T_{\text {sky }} \propto v^{\gamma}\right.$ with $\left.\gamma \sim 2.5\right)$. It means that in LOFAR wide-band observations, the noise in the low-frequency sub-bands will be higher than that in the highfrequency sub-bands. In the cases of observations dominated by the radiation from the Galaxy, Eq. 6 must be adapted to take into account the different uncertainties in each sub-band.

Thermal noise from the sky brightness temperature translates into a Gaussian-like noise in the real and imaginary parts of the visibilities, with a value of $\sigma$ proportional to the equivalent flux density of the system, which is in turn proportional to the total (i.e., receivers plus source) temperature (e.g., Thomson, et al. 1991). If the observed sources are strong, the noise in the amplitudes and phases can also be approximated as being 


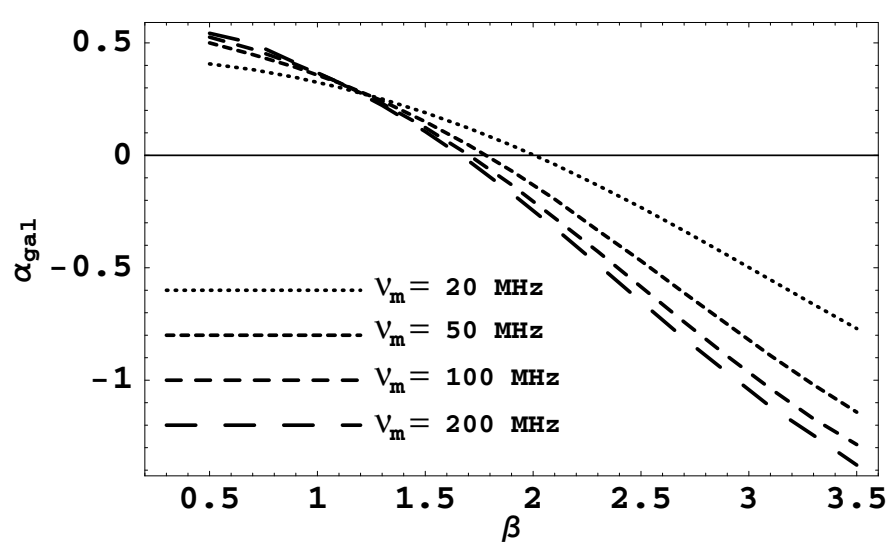

Fig. 7. Optimum value of $\alpha$ that minimizes the $\sigma(K)$ given in Eq. (18) for different values of the minimum frequency, $v_{\mathrm{m}}$. These values of $\alpha_{\mathrm{gal}}$ have been computed assuming that the Galaxy radiation dominates the noise of the visibilities. A line at $\alpha_{\text {gal }}=0$ is also shown for clarity.

Gaussian-like, with a $\sigma$ proportional to that of the real and imaginary parts of the visibilities. If we take this approximation into account and assume that the galactic radiation dominates the system temperature (i.e., $T_{\text {sys }} \sim T_{\text {sky }}$ ), then the uncertainty in the visibility phase of the $i$ th sub-band is $\sigma_{i} \propto v_{i}^{-5}$ and Eq. (6) becomes

$\sigma(K) \propto \frac{\sum v_{i}^{5}}{\sum v_{i}^{3} \sum v_{i}^{5}-\left(\sum v_{i}^{4}\right)^{2}}$.

The values of $\alpha$ in Eq. (10) that minimize the $\sigma(K)$ given by Eq. (18) (we call these values $\alpha_{\text {gal }}$ ) are shown in Fig. 7 as a function of the normalized bandwidth, $\beta$. For small values of $\beta$, we find that $\alpha_{\text {gal }}$ is positive. It means that the noise in the phases at the lower frequencies is such high, that a better sampling of the high-frequency region of the band yields more precise estimates of the ionospheric dispersion. However, this effect is less important for wider bands and/or higher minimum frequencies, as it can be seen in Fig. 7. If the band is wide enough (for instance, $\beta>1.7$ for a minimum frequency of $200 \mathrm{MHz}$ ) the optimum value $\alpha_{\text {gal }}$ is negative. The $20-90 \mathrm{MHz}$ band of LOFAR corresponds to a value of $\beta$ as large as 3.5 , for which we find $\alpha_{\text {gal }}=-0.8\left(\right.$ setting $\left.\nu_{\mathrm{m}}=20 \mathrm{MHz}\right)$.

\section{Summary}

We studied the achievable precision in the estimate of the ionospheric dispersion, the ambiguity of the group delay, the dynamic range of the correlated fringes, and the precision in the estimate of the source spectral index in low-frequency and wide-band interferometric observations for four different distributions of the sub-bands through the total bandwidth of the detectors: constant spacing between sub-bands, random spacings, spacings based on a power-law distribution, and spacings based on Golomb rulers.

For a large number of sub-bands, spacings based on Golomb rulers give the most precise estimates of dispersive effects and the highest dynamic ranges of the fringes. Spacings based on the power-law distribution give similar (but slightly worse) results, although the results are better than those from the Golomb rulers for a smaller numbers of sub-bands. Random distributions of sub-bands result in relatively large dynamic ranges of the fringes, but the estimate of dispersive effects through the band is worse. A constant spacing of the sub-bands results in very bad dynamic ranges of the fringes, but the estimates of dispersive effects have a precision similar to that obtained with the power-law distribution.

From all combinations of the number of sub-bands and the total covered bandwidth, the power-law distribution (with $\alpha$ given by Eq. (12)) gives the best compromise between homogeneity in the sampling of the bandwidth, precision in the estimate of the ionsopheric dispersive effects, dynamic range of the correlated fringes, and ambiguity of the group delay. Therefore, this would be the preferable sub-band distribution to use in lowfrequency (wide-band) interferometric observations.

Finally, we study how the power-law distribution that optimally samples the ionospheric dispersion is affected in the cases of 1) observing frequencies close to the plasma frequency of the ionospheric electrons; 2) chromatic effects in the structure of the sources; and 3) non-negligible noise coming from the Galaxy radiation.

Acknowledgements. The author is a fellow of the Alexander von Humboldt Foundation in Germany. The author is very thankful to Eduardo Ros and the anonymous referee for their useful comments and suggestions to improve this paper. The author also acknowledges the collaboration of Nicolás Martí-Dunca during the preparation of this paper.

\section{References}

Atkinson, M. D., Santoro, N., \& Urrutia, J. 1986, IEEE Transactions on Communications, COM-34, 614

Blandorf, R. D., \& Königl, A. 1979, ApJ, 232, 34

Cohen, A. S., \& Röttgering, H. J. A. 2009, AJ, 138, 439

Intema, H. T., van der Tol, S., Cotton, W. D., et al. 2009, A\&A, 501, 1185

Mioduszewski, A. J., \& Kogan, L. 2004, AIPS Memo 110

Pacholczyk, A. G. 1970, Radio Astrophysics, Freeman, San Francisco

Petrachenko, B. 2008, IVS Memorandum 2008-005v01

Porcas, R. W. 2009, A\&A, 505, L1

Ros, E., Marcaide, J. M., Guirado, J. C., Sardón, E., \& Shapiro, I. I. 2000, A\&A, 356,357

Thomson, A. R., Moran, J. M., \& Swenson, G. W. 1991, Interferometry and Synthesis in Radio Astronomy, Krieger Publ. Corp., Florida

Todorova, S., Hobiger, T., \& Schuh, H. 2006, 36th COSPAR Scientific Assembly, 36, 2405

de Vos, M., Gunst, A. W., \& Nijboer, R. 2009, IEEE Proceedings, 97, 1431 